\title{
Construction and analysis of protein-protein interaction networks based on proteomics data of prostate cancer
}

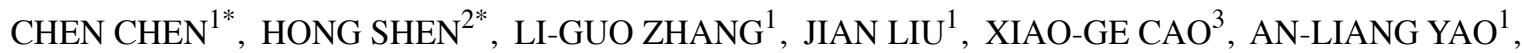 \\ SHAO-SAN KANG ${ }^{1}$, WEI-XING GAO ${ }^{1}$, HUI HAN ${ }^{1}$, FENG-HONG CAO ${ }^{1}$ and ZHI-GUO LI ${ }^{4}$ \\ ${ }^{1}$ Department of Urology, North China University of Science and Technology Affiliated Hospital; \\ ${ }^{2}$ Department of Modern Technology and Education Center, North China University of Science and Technology \\ and International Science and Technology Cooperation Base of Geriatric Medicine, Tangshan, Hebei; ${ }^{3}$ Tianjin Binhai New \\ Area Hangu No. 1 High School, Tianjin 300480; ${ }^{4}$ Department of Medical Research Center, \\ North China University of Science and Technology and International Science and Technology \\ Cooperation Base of Geriatric Medicine, Tangshan, Hebei 063000, P.R. China
}

Received October 16, 2015; Accepted April 15, 2016

DOI: 10.3892/ijmm.2016.2577

\begin{abstract}
Currently, using human prostate cancer (PCa) tissue samples to conduct proteomics research has generated a large amount of data; however, only a very small amount has been thoroughly investigated. In this study, we manually carried out the mining of the full text of proteomics literature that involved comparisons between $\mathrm{PCa}$ and normal or benign tissue and identified 41 differentially expressed proteins verified or reported more than 2 times from different research studies. We regarded these proteins as seed proteins to construct a protein-protein interaction (PPI) network. The extended network included one giant network, which consisted of 1,264 nodes connected via 1,744 edges, and 3 small separate components. The backbone network was then constructed, which was derived from key nodes and the subnetwork consisting of the shortest path between seed proteins. Topological analyses of these networks were conducted to identify proteins essential for the genesis of $\mathrm{PCa}$. Solute carrier family 2 (facilitated glucose transporter), member 4 (SLC2A4) had the highest closeness centrality located in the center of each network, and the highest betweenness centrality and largest degree in the backbone network. Tubulin, beta 2C (TUBB2C)
\end{abstract}

Correspondence to: Dr Feng-Hong Cao, Department of Urology, North China University of Science and Technology Affiliated Hospital, 73 South Construction Road, Tangshan, Hebei 063000, P.R. China E-mail: caofenghong@163.com

Dr Zhi-Guo Li, Medical Research Center, North China University of Science and Technology and International Science and Technology Cooperation Base of Geriatric Medicine, 57 South Construction Road, Tangshan, Hebei 063000, P.R. China

E-mail: lzg1017@163.com

*Contributed equally

Key words: network, prostate cancer, protein-protein interaction, proteomics had the largest degree in the giant network and subnetwork. In addition, using module analysis of the whole PPI network, we obtained a densely connected region. Functional annotation indicated that the Ras protein signal transduction biological process, mitogen-activated protein kinase (MAPK), neurotrophin and the gonadotropin-releasing hormone $(\mathrm{GnRH})$ signaling pathway may play an important role in the genesis and development of PCa. Further investigation of the SLC2A4, TUBB2C proteins, and these biological processes and pathways may therefore provide a potential target for the diagnosis and treatment of PCa.

\section{Introduction}

In recent decades, prostate cancer (PCa) has become one of the most common types of cancers in Europe and in the United States (1). The American Cancer Society predicted that there would be 220,800 new cases of PCa with 27,540 deaths in 2015 (2). The pathogeny and risk factors of PCa are complex and not yet fully understood. Thus, it appears to be urgent and meaningful to further investigate the tumor-associated proteins and PCa pathogenesis. The rise of omics technologies in recent years and their use in PCa research have delivered a number of novel potential biomarkers for PCa (3). Among the available technologies, the ongoing improvements in proteomics technologies have resulted in increased information on protein behavior during cellular processes. The exponential accumulation of proteomics data has the potential to improve the treatment and prognosis of PCa by shedding light on the most important events that regulate prostate cells under normal and pathophysiological conditions (4). The standard proteomics experimental strategies are designed to compare proteomics signatures of cells in normal and anomalous states. As a result, a set of proteins with differential expression levels as a consequence of the different states of the cells is delivered. To fully understand the cellular machinery that leads to the different states, simply listing these identified proteins is not sufficient. As protein-protein interactions (PPI) are the key mechanisms of almost all biological processes, the characterization of all possible interactions connecting the iden- 
tified proteins is required (5). At the same time, using PPI to form a molecular regulatory network, which includes key nodes and signal transduction pathways, not only helps to further understand the biological processes from a systems perspective, but can also be used to predict and evaluate the corresponding treatments, providing a theoretical basis for the search for novel drug targets (6). Nevertheless, limited research associated with protein interactions specific to PCa proteomics-related proteins has been conducted. Therefore, to put our proteomics data in a biological context, we used a systems biology approach (the platform of PCa-related protein networks based on the available proteomics literature data) as a rational strategy for the identification of novel specific markers and novel therapeutic targets.

Network science is gradually altering our view of cell biology by offering unforeseen possibilities to understand the internal organization of a cell (7). Rather than the traditional approach of studying individual proteins or genes, a systematic investigation of PCa proteins in the human PPI network may provide important biological information for uncovering the molecular mechanisms of PCa (8). In recent years, there have been a number of successful studies that have found important target genes and markers by constructing and analyzing the protein interaction networks associated with diseases. In 2011, Lee et al constructed PPI networks of abnormally expressed genes for schizophrenia, bipolar disease and major depression, and identified several disease markers, such as strawberry notch homolog 2 (Drosophila) (SBNO2) for schizophrenia, SEC24 homolog C, COPII coat complex component (SEC24C) for bipolar disorder and serrate, RNA effector molecule (SRRT) for major depression (9). In April 2013, Ran et al constructed and analyzed PPI networks for essential hypertension (EH), and suggested that blood pressure variations related to $\mathrm{EH}$ are orchestrated by an integrated PPI network with the protein encoded by the nitric oxide synthase 3 (endothelial cell) (NOS3) gene (10). Recently, in August 2014, Rakshit et al constructed PPI networks based on gene expression profiles of Parkinson's disease (PD) and identified 37 network biomarkers that can be used as potential therapeutic targets for PD applications developments (7).

In this study, we mined the differentially expressed proteins (DEPs) verified or reported more than 2 times in the proteomics literature of PCa. The DEPs were regarded as seed proteins to construct an extended PPI network, which not only consisted of the seed proteins, but also their direct PPI neighbors and the interactions between these proteins. Topological analyses were performed to determine the significant network biomarkers. The association of these biomarkers with the genesis of PCa was investigated. The backbone network constructed of key nodes and the subnetwork of the shortest paths, as well as the densely connected region, were also investigated. Thus, the findings of this study may provide insight into the potential targets for developing novel treatment strategies for PCa.

\section{Data collection and methods}

Mining proteomics literature of PCa and screening criteria. We used the PubMed Database as the main source for literature retrieval and adopted an advanced search option by inputting '(prostate cancer[Title]) AND Proteomics' for literature before
July 26th, 2015. The search was limited to publications in English. The inclusion criteria were the following: i) studies on the human species. ii) Studies using PCa tissue samples, including samples obtained by prostate needle biopsies and radical prostatectomy. The key to a more effective diagnosis, prognosis and therapeutic management of PCa could lie in the direct analysis of cancer tissue. Prostate tissue has the advantage over other biomaterials, that in addition to being a rich source of potential $\mathrm{PCa}$ biomarkers, it offers the possibility to clarify the mechanisms at the basis of the transformation of a normal prostate cell to a tumor cell and subsequent progression to a metastatic state (11). iii) Studies involved in the comparison between the tumor and normal or benign tissues, including PCa and benign prostatic hyperplasia (BPH), PCa and adjacent benign tissues or PCa and normal tissues. Exclusion criteria were the following: i) Studies on non-human tissue. ii) Studies on the PCa cell lines. Human tumor tissues and cell lines cultured in vitro are each valuable resources. However, the in vitro environment may cause changes in protein expression level of PCa cells. Therefore, in this study, we were limited to human tumor tissues. iii) Studies on samples of biological fluids, including serum, plasma, urine, seminal plasma and expressed prostatic secretions. iv) Studies only involved in tumor aggressiveness or metastasis. v) Studies on the effects of certain interventions on the protein expression profiles. vi) Studies which did not supply names or accession numbers of DEPs. vii) Literature reviews.

Extraction of DEPs associated with PCa proteomics. To prevent the omission of DEPs, we manually carried out the evaluation of publications in line with the above conditions; a total of 175 DEPs was extracted. The DEPs were turned into corresponding official gene symbols with the Protein Information Resource (PIR, Georgetown University Medical Center, Washington, DC, USA). The PIR is an integrated public bioinformatics resource to support genomic, proteomic and systems biology research and scientific studies (12).

Since proteomics technologies use complex instrumentation and costly consumables, the majority of investigations have a small sample size, and this, coupled with the relatively low reliability of the proteomics technology itself, leads to the reliability of the final results being questionable. We believed that if different proteomics studies have obtained the same DEPs, the reliability of the proteins associated with PCa would be greatly enhanced. Therefore, to improve the accuracy and reliability of the DEPs to the maximum extent, we extracted the DEPs verified or reported more than 2 times from different proteomics studies as seed proteins. The count criteria were as follows: i) If in one article several DEPs have the same official gene symbol, that was counted as once. ii) If in one article some DEPs were selected by the proteomics methods as the protein of interest to be further experimentally verified (by western blot analysis or immunohistochemistry), that was counted as twice. A total of 41 DEPs were obtained (Table I) as the seed proteins of the construction of the PCa proteomics PPI network.

Construction of the PPI network and extracting the giant component from the extended network. To avoid the loss of protein interactions using a single database, we used a combination of multiple databases to construct the network. Although the protein interactions in the different databases 
Table I. List of the 41 differentially expressed proteins between prostate cancer and normal or benign tissues by literature mining and screening the reported frequencies.

\begin{tabular}{|c|c|c|c|}
\hline Gene ID & Symbol (Refs.) & Description & $\begin{array}{l}\text { Reported } \\
\text { frequency }\end{array}$ \\
\hline 1674 & DES $(11,48-52)$ & Desmin & 6 \\
\hline 3329 & HSPD1 $(11,48,50,51,53)$ & Heat shock $60 \mathrm{kDa}$ protein 1 (chaperonin) & 5 \\
\hline 3309 & HSPA5 $(48,50,51)$ & Heat shock $70 \mathrm{kDa}$ protein 5 (glucose-regulated protein, $78 \mathrm{kDa}$ ) & 3 \\
\hline 3875 & KRT18 $(11,50,51)$ & Keratin 18 , type I & 3 \\
\hline 3856 & KRT8 $(11,50,51)$ & Keratin 8, type II & 3 \\
\hline 7414 & $\operatorname{VCL}(48,50,54)$ & Vinculin & 3 \\
\hline 55 & $\operatorname{ACPP}(50,53)$ & Acid phosphatase, prostate & 2 \\
\hline 213 & $\operatorname{ALB}(11,51)$ & Albumin & 2 \\
\hline 308 & ANXA5 $(50,52)$ & Annexin A5 & 2 \\
\hline 392 & ARHGAP1 $(11,50)$ & Rho GTPase activating protein 1 & 2 \\
\hline 396 & ARHGDIA $(50,51)$ & Rho GDP dissociation inhibitor (GDI) $\alpha$ & 2 \\
\hline 563 & AZGP1 $(11,50)$ & alpha-2-glycoprotein 1, zinc-binding & 2 \\
\hline 822 & CAPG $(50,54)$ & Capping protein (actin filament), gelsolin-like & 2 \\
\hline 1152 & CKB $(51,52)$ & Creatine kinase, brain & 2 \\
\hline 30846 & EHD2 $(11,48)$ & EH-domain containing 2 & 2 \\
\hline 2023 & ENO1 $(50,53)$ & Enolase $1,(\alpha)$ & 2 \\
\hline 2266 & FGG $(50,51)$ & Fibrinogen gamma chain & 2 \\
\hline 2288 & FKBP4 $(11,50)$ & FK506 binding protein $4,59 \mathrm{kDa}$ & 2 \\
\hline 2638 & $\mathrm{GC}(11,50)$ & Group-specific component (vitamin D binding protein) & 2 \\
\hline 2934 & $\operatorname{GSN}(48,50)$ & Gelsolin & 2 \\
\hline 2947 & $\operatorname{GSTM} 3(50,54)$ & Glutathione S-transferase mu 3 (brain) & 2 \\
\hline 2950 & GSTP1 $(50,54)$ & Glutathione S-transferase pi 1 & 2 \\
\hline 3187 & HNRPH1 $(48,50)$ & Heterogeneous nuclear ribonucleoprotein H1 (H) & 2 \\
\hline 3313 & HSPA9 $(48,51)$ & Heat shock $70 \mathrm{kDa}$ protein 9 (mortalin) & 2 \\
\hline 3315 & HSPB1 $(48,50)$ & Heat shock $27 \mathrm{kDa}$ protein 1 & 2 \\
\hline 3848 & KRT1 $(11,50)$ & Keratin 1, type II & 2 \\
\hline 3880 & KRT19 $(50,51)$ & Keratin 19 , type I & 2 \\
\hline 5034 & P4HB $(50,51)$ & Prolyl 4-hydroxylase, beta polypeptide & 2 \\
\hline 5245 & PHB $(50,51)$ & Prohibitin & 2 \\
\hline 7052 & TGM2 $(49,50)$ & Transglutaminase 2 & 2 \\
\hline 7163 & TPD52 $(48,50)$ & Tumor protein D52 & 2 \\
\hline 7168 & TPM1 $(49,52)$ & Tropomyosin $1(\alpha)$ & 2 \\
\hline 10383 & TUBB2C $(50,51)$ & Tubulin, beta $2 \mathrm{C}$ & 2 \\
\hline 7431 & VIM $(48,52)$ & Vimentin & 2 \\
\hline 3615 & IMPDH2 $^{\text {a }}(54)$ & IMP (inosine 5'-monophosphate) dehydrogenase 2 & 2 \\
\hline 64087 & $\operatorname{MCCC}^{\mathrm{a}}(54)$ & Methylcrotonoyl-CoA carboxylase $2(\beta)$ & 2 \\
\hline 10631 & $\operatorname{POSTN}^{\mathrm{a}}(48)$ & Periostin, osteoblast specific factor & 2 \\
\hline 5500 & $\operatorname{PPP}_{1 C B}{ }^{a}(11)$ & Protein phosphatase 1 , catalytic subunit, beta isozyme & 2 \\
\hline 5694 & $\operatorname{PSMB6}^{\mathrm{a}}(11)$ & Proteasome (prosome, macropain) subunit, beta type, 6 & 2 \\
\hline 10131 & $\mathrm{TRAP}^{\mathrm{a}}(54)$ & TNF receptor-associated protein 1 & 2 \\
\hline 7334 & $\mathrm{UBE}_{2} \mathrm{~N}^{\mathrm{a}}(11)$ & Ubiquitin-conjugating enzyme E2N & 2 \\
\hline
\end{tabular}

${ }^{a}$ Gene symbol with 'a' indicates that the protein only appeared in one article, but was given further experimental (western blot analysis and immunohistochemistry) validation as a protein of interest. Apart from those labeled with 'a, , the remaining proteins were at found in at least two studies.

largely overlap, the databases are complementary (13). We made use of the POINeT bioinformatics tool to form the human PPI network. POINeT is currently a relatively popular construction tool that integrates PPI information collected by various protein databases, including the Database of Interacting Proteins (DIP), Molecular INTeraction database (MINT), Biomolecular Interaction Network Database (BIND), Human Protein Reference Database (HPRD), Mammalian 
Protein-Protein Interaction Database (MIPS), MIPS Comprehensive Yeast Genome Database (CYGD), Biological General Repository for Interaction Datasets (BioGRID) and NCBI database (14). The PPI networks constructed by POINeT were then visualized using Cytoscape 3.2.1, which is a software package available on the internet for biological network visualization, data integration and interactive network generation (15). In this study, the extended network included a giant component and 3 small separate components derived from 3 seed proteins. This study aimed to explore the mechanisms responsible for $\mathrm{PCa}$ at the system level; the major central nodes must be in the giant network as the 3 small separate components consisted of a very small number of nodes, so only the giant network and its parameters related to the network theory had been analyzed or processed. To analyze and process the giant network conveniently, we extracted it from the extended network.

Topological analysis of the protein interaction network. The molecular organization can be visualized as a network of differentially connected nodes. Each node stands for a protein and the edges represent dynamic interactions. Nodes thereby receive input and output values as mathematical functions (16). In the theory of the network, the connectivity degree $(\mathrm{k})$, betweenness centrality (BC) and closeness centrality (CC) value of nodes are three fundamental parameters that are usually adopted to evaluate the nodes in a network (17). The degree, defined as the number of interacting partner proteins, is the most basic characteristic of a node. The $\mathrm{BC}$ value is the fraction of the number of shortest paths that pass through each node in a network, which measures how often the node is located on the shortest paths between other nodes (18). The shortest path is the path containing the least number of vertices between two vertices in a network. A node with a higher $\mathrm{BC}$ value indicates that it has more influence over the information flow in the network. Therefore, BC values are generally useful indicators to detect bottlenecks in a network. The $\mathrm{CC}$ value is the inverse of the average length of the shortest paths to/from all other nodes in the graph and measures how close a node is to other nodes. The node with the highest $\mathrm{CC}$ value is usually the topological center of the network (18). In this study, Cytoscape 3.2.1 (15) was used to calculate the properties of the nodes and perform measurements under default parameters.

Creation of the backbone network of the PPI network for PCa. In the theory of the graph, proteins with high $\mathrm{BC}$ values are usually thought to be bottlenecks controlling the information flow in the transportation network (10). We set the critical node with a high $\mathrm{BC}$ value at $5 \%$ of the total nodes in the network. The proteins with a higher $\mathrm{BC}$ value and the links between them will make up a backbone network. Thus, we extracted the proteins with the top 5\% of BC values and the links between them from the PPI network for PCa to create a backbone network.

Construction of a subnetwork consisting of all of the shortest paths between the seed proteins. Even in the giant network, there are a few pairs of seed proteins that are not connected directly. To construct a subnetwork in which all proteins associated with $\mathrm{PCa}$ are connected directly or indirectly with the minimum number of nodes, we found all the shortest paths between every pair of seed proteins. Pesca3.0 (19), a plug-in for Cytoscape, was used to calculate the shortest paths between the
41 seed proteins. The subnetwork consists of all nodes in these shortest paths. The subnetwork indicates the possible minimal number of connections among the 41 seed proteins responsible for the genesis of PCa.

Identification of densely connected regions in the PPI network. Biological networks are likely comprised of several subnetworks or functional modules contributing to various diverse biological processes. A node may have negligible impact on the global network or global properties, yet is influential on a subnetwork with specific functionality (14). Therefore, we used Mcode1.4.1 (20) (a plug-in for Cytoscape) to cluster the whole network to identify densely connected regions. The module division parameters were as follows: degree cut-off, 2; node score cut-off, 0.2; k-core, 2; and max depth, 100. After clustering the PPI network, function annotation of the nodes located in the cluster was performed by DAVID Bioinformatics Resources 6.7 (the Database for Annotation, Visualization and Integrated Discovery, from National Institute of Allergy and Infectious Diseases, NIH, USA) (21).

The function annotation includes GO (Gene Ontology) analysis and KEGG (Kyoto Encyclopedia of Genes and Genomes) pathways analysis. GO categories analysis provides a common descriptive framework to functionally annotate and classify gene sets. GO categories are organized into 3 groups: biological process, cellular component and molecular function. KEGG pathways bring together the molecular interactions and the reaction networks through an artificial pathway diagram.

The Benjamini method was used to control the false discovery rate (FDR) to correct the P-value. The Benjamini method is useful in large-scale multiple testing problems based on discrete test statistics and derive its basic asymptotic (as the number of hypotheses tends to infinity) properties, subsuming earlier results.

\section{Results}

PPI network. The extended network includes one giant network and 3 separated small components that are derived from the seed proteins, alpha-2-glycoprotein 1, zinc-binding (AZGP1), capping protein (actin filament), gelsolin-like (CAPG) and periostin, osteoblast specific factor (POSTN) (Fig. 1). The giant network consisted of 1,264 nodes connected via 1,744 edges (Fig. 2). The backbone network consisted of 63 nodes connected via 186 edges (Fig. 3). We compared the measurement parameters of the network between the giant network and the backbone network (Table II). The largest degree in the giant network was 174 , while the average degree was 2.759 . This network is characterized by a small number of highly connected nodes, while most of the other nodes have few connections, which is the classical character of a PPI network (22).

Key nodes in the PPI network. In this study, the nodes with a large degree or high BC were viewed as key nodes, and $5 \%$ of the total nodes set of the network was used as the critical point of large degree and high BC nodes. Of the 1,264 total nodes, 63 nodes had a high $\mathrm{BC}$ value, 58 nodes had a large degree and 50 nodes were selected with a high $\mathrm{BC}$ value and large degree. To distinguish the different roles of these key nodes in the network, they were highlighted with different colors and sizes. 


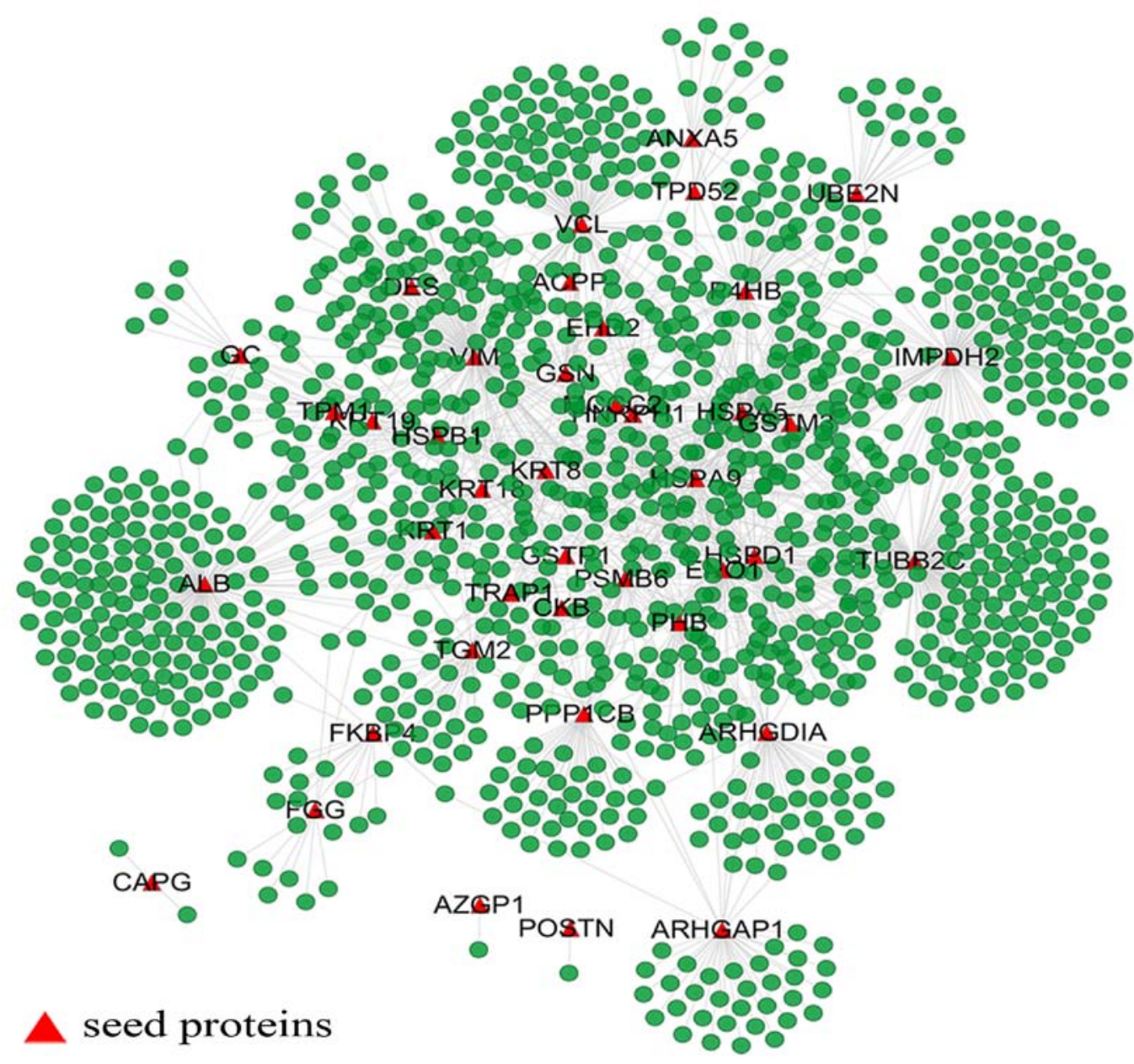

Figure 1. Overview of the extended network. The extended network includes one giant network and 3 separate small components which are derived, respectively, from the seed proteins, AZGP1, CAPG and POSTN. Nodes with a red triangular shape are the seed proteins shown in Table I, the rest are their neighbors.

Table II. The general measurements for each network.

\begin{tabular}{llcc}
\hline Parameter & $\begin{array}{c}\text { Giant } \\
\text { network }\end{array}$ & $\begin{array}{c}\text { Backbone } \\
\text { network }\end{array}$ & Subnetwork \\
\hline No. of nodes & 1,264 & 63 & 302 \\
Average degree & 2.759 & 5.905 & 5.093 \\
Largest degree & 174 & 17 & 72 \\
$\begin{array}{l}\text { Diameter } \\
\text { Mean shortest }\end{array}$ & 7 & 5 & 5 \\
path length & 3.859 & 2.675 & 3.145 \\
\hline
\end{tabular}

The size of the nodes corresponded to their BC values (Fig. 2). Tubulin, beta 2C (TUBB2C) is a hub protein with the largest degree and second highest $\mathrm{BC}$ value, while albumin (ALB) is a bottleneck protein with the highest $\mathrm{BC}$ value and the second largest degree. Solute carrier family 2 (facilitated glucose transporter), member 4 (SLC2A4) has the highest CC value, which indicates that SLC2A4 is located at the center of the network.

Backbone network of the PPI network. The backbone network consisted from 63 nodes with a high $\mathrm{BC}$ value, the size of which corresponds to their $\mathrm{BC}$ value and the 186 links between them (Fig. 3). SLC2A4 was located at the center of the backbone network with the highest CC value. SLC2A4 also had the largest degree and the highest $\mathrm{BC}$ value; thus, it controls the information flow in the backbone network. SLC2A4 has 17 neighbors: heat shock $70 \mathrm{kDa}$ protein (HSPA)5, TUBB2C, vimentin (VIM), HSPA9, heat shock $60 \mathrm{kDa}$ protein 1 (HSPD1), IMP (inosine 5'-monophosphate) dehydrogenase 2 (IMPDH2), prolyl 4-hydroxylase, beta polypeptide (P4HB), enolase 1 (ENO1), glutathione S-transferase Pi 1 (GSTP1), heat shock $27 \mathrm{kDa}$ protein 1 (HSPB1), protein phosphatase 1, catalytic subunit, beta isozyme (PPP1CB), keratin 8, type II (KRT8), ubiquitin-conjugating enzyme E2N (UBE2N), Rho GDP dissociation inhibitor (GDI) Alpha (ARHGDIA), proteasome subunit beta 6 (PSMB6), desmin (DES) and EH-domain containing 2 (EHD2). The details of the other proteins in the backbone network are not presented here.

Subnetwork consisting of the shortest paths between the seed proteins. The subnetwork consisted of 302 nodes and 769 edges (Fig. 4). We found that TUBB2C had the highest $\mathrm{BC}$ value and the largest degree. SLC2A4 was also located at the center of the subnetwork and had the highest $\mathrm{CC}$ value. This is consistent with the results of the giant network and 


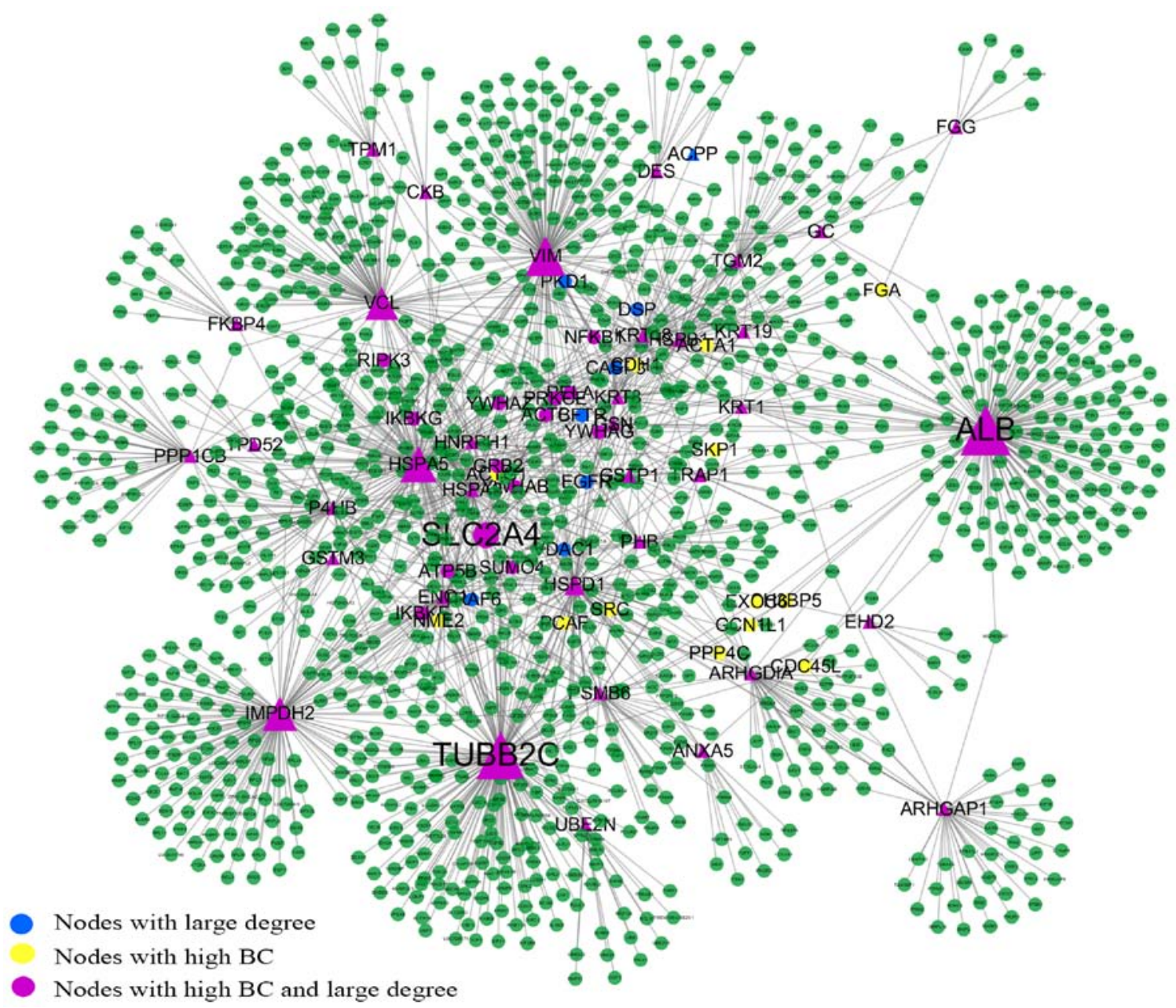

Figure 2. Topology of the giant network. The giant network extracted from the extended network is the biggest component in the extended network. It consisted of 1,264 nodes and 1,744 edges. Key nodes in the giant network are highlighted in different colors. Nodes with a triangular shape are the seed proteins. The size of the nodes corresponds to their BC values. SLC2A4 is located at the center of the giant network. BC, betweenness centrality.

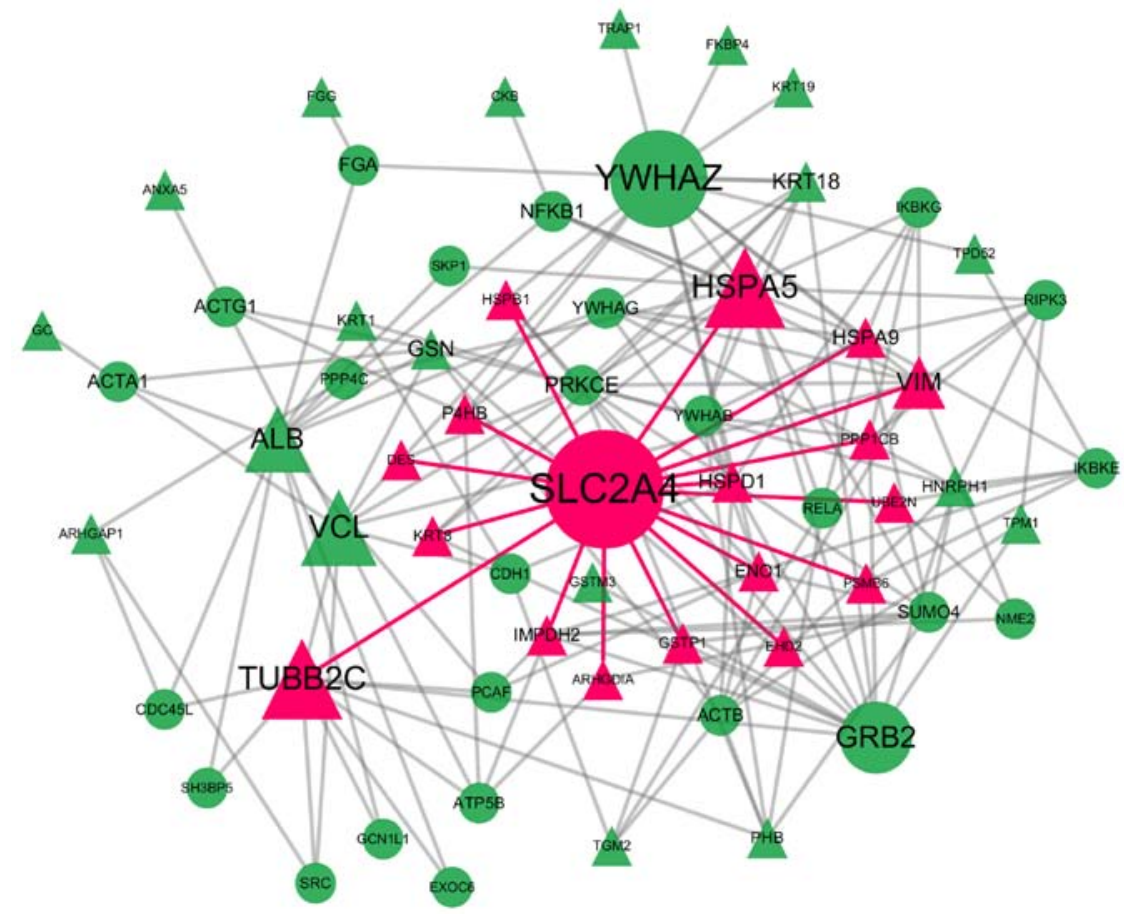

Figure 3. Topology of the backbone network. The backbone network consisted of 63 nodes with a high BC value and 186 edges. The size of the nodes corresponds to their BC values. Nodes marked with red are the 17 neighbors of SLC2A4. BC, betweenness centrality. 


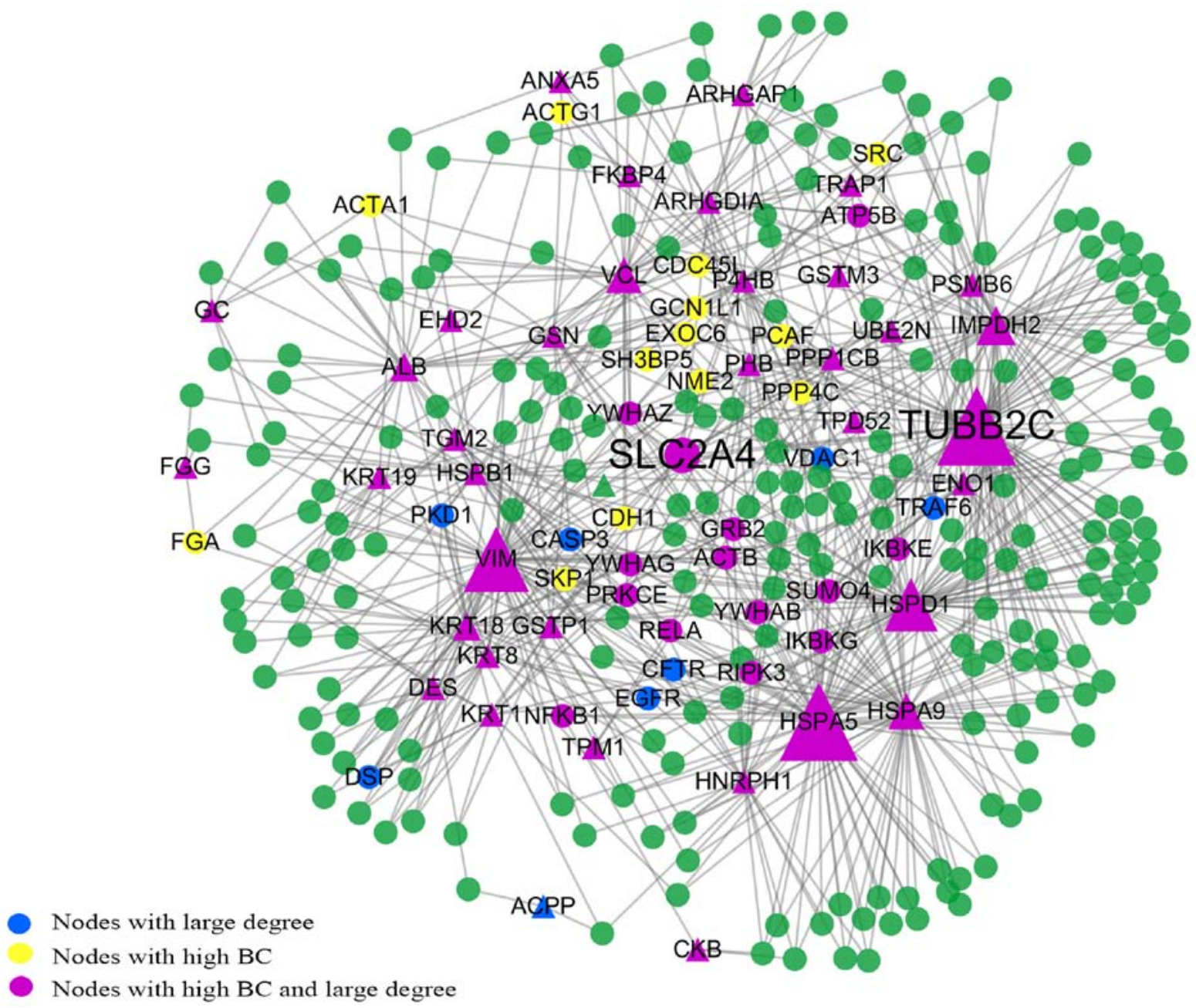

Figure 4. The subnetwork consisting of all of the shortest paths between the 41 seed proteins. The subnetwork consisted of 302 nodes and 769 edges. The size of the nodes corresponds to their BC values. SLC2A4 is located at the center of the subnetwork. TUBB2C has the highest BC value and the largest degree. $\mathrm{BC}$, betweenness centrality.

backbone network. In these 302 points, apart from AZGP1, CAPG and POSTN which are related to 3 separate small components, the rest of the seed proteins are all among these points. We determined that the top $63 \mathrm{BC}$ nodes in this subnetwork coincided well with the 63 nodes in the backbone network. There are only 16 proteins which are not in the list of the 63 nodes with large $\mathrm{BC}$ values in the giant network. These are epidermal growth factor receptor (EGFR), cystic fibrosis transmembrane conductance regulator (ATP-binding cassette subfamily C, member 7) (CFTR), immunoglobulin heavy constant mu (IGHM), caspase-3 (CASP3), GINS complex subunit 2 (Psf2 homolog) (GINS2), exocyst complex component 5 (EXOC5), voltage-dependent anion channel 1 (VDAC1), nuclear factor of kappa light polypeptide gene enhancer in B-cells inhibitor, alpha (NFKBIA), tubulin, gamma 1 (TUBG1), tumor necrosis factor receptor superfamily, member 1A (TNFRSF1A), GDP-mannose pyrophosphorylase B (GMPPB), TNF receptor-associated factor 6, E3 ubiquitin protein ligase (TRAF6), cyclindependent kinase 2 (CDK2), heterogeneous nuclear ribonucleoprotein $\mathrm{K}$ (HNRPK), eukaryotic translation initiation factor 4 gamma, 1 (EIF4G1) and 3-hydroxy-3-methylglutaryl-CoA synthase 1 (soluble) (HMGCS1).
Densely connected region in the constructed PPI network for $P C a$. Through M-code module analysis, we found a densely connected region with keratin 18, type I (KRT18), growth factor receptor-bound protein 2 (GRB2), EGFR, HSPA5 and KRT8 as the main nodes (Fig. 5). Among these, KRT18, HSPA5 and KRT8 were seed proteins. Module function annotational by DAVID revealed that the module was mainly enriched in the apoptosis and Ras protein signal transduction biological processes (Table III). The KEGG pathway analysis revealed the involvment of the MAPK signaling pathway, neurotrophin signaling pathway, GnRH signaling pathway, colorectal cancer and non-small cell lung cancer (Table IV).

\section{Discussion}

Currently, PCa proteomics has generated a large amount of data; however, only a very small amount has been thoroughly investigated. Mining these DEPs and building a PPI network could be regarded as an effective way to explore the biological significance behind it. Mining of the proteomics data may further reveal novel pathogenic mechanisms by contributing to the characterization and understanding of biological processes and their aberrant functions in $\mathrm{PCa}$; it may also provide a 


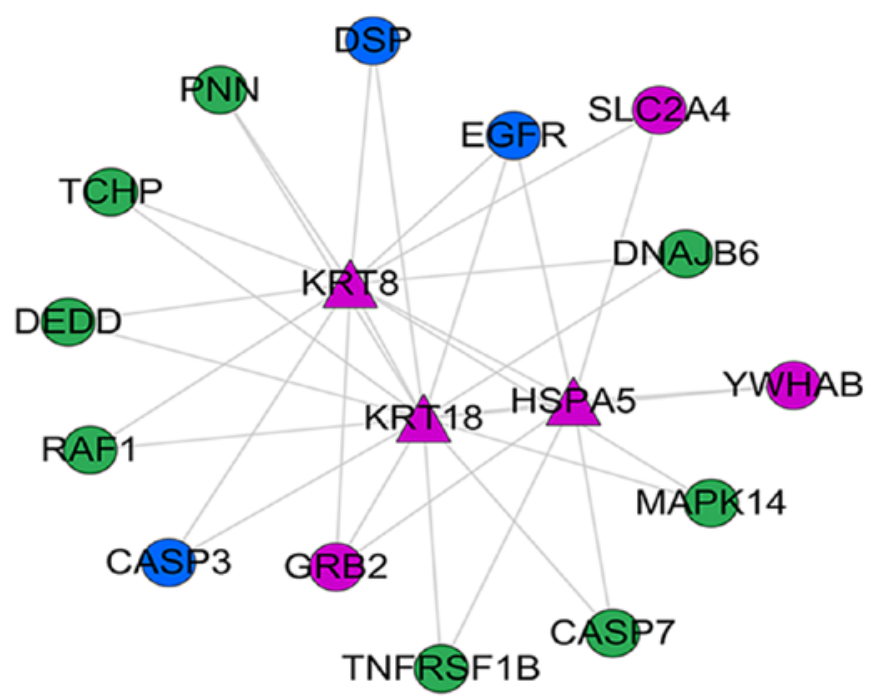

Nodes with large degree

Nodes with high BC and large degree

Figure 5. The significant modules in the whole extended network.

Table III. Gene Ontology (GO) functional enrichment analysis of the densely connected region with the threshold of $\mathrm{P}<0.05$.

\begin{tabular}{lllcrr}
\hline Category & GO ID & \multicolumn{1}{c}{ Term } & Count & P-value & Size \\
\hline BP & GO:0006915 & Apoptosis & 8 & $6.4 \mathrm{E}-4$ & 602 \\
BP & GO:0007265 & Ras protein signal transduction & 4 & $9.8 \mathrm{E}-3$ & 105 \\
BP & GO:0042981 & Regulation of apoptosis & 7 & $1.1 \mathrm{E}-2$ & 804 \\
\hline
\end{tabular}

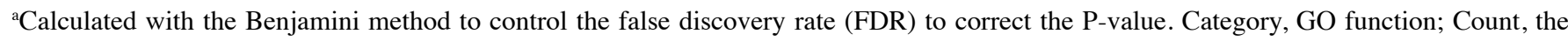
number of proteins; Size, the total number of genes in the GO BP. BP, biological process.

Table IV. KEGG pathway enrichment analysis of the densely connected region with the threshold of $\mathrm{P}<0.05$.

\begin{tabular}{lcccc}
\hline KEGG pathway & KEGG entry & Count & P-value $^{\mathrm{a}}$ & Size \\
\hline $\begin{array}{l}\text { MAPK signaling } \\
\text { pathway }\end{array}$ & hsa04010 & 5 & $3.5 \mathrm{E}-2$ & 267 \\
$\begin{array}{l}\text { Neurotrophin } \\
\text { signaling pathway }\end{array}$ & hsa04722 & 4 & $2.9 \mathrm{E}-2$ & 124 \\
$\begin{array}{l}\text { GnRH signaling } \\
\text { pathway }\end{array}$ & hsa04912 & 4 & $2.9 \mathrm{E}-2$ & 98 \\
$\begin{array}{l}\text { Colorectal cancer } \\
\begin{array}{l}\text { Non-small cell } \\
\text { lung cancer }\end{array}\end{array}$ & hsa05210 & 4 & $3.7 \mathrm{E}-2$ & 84 \\
\hline
\end{tabular}

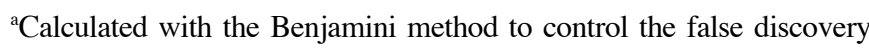
rate (FDR) to correct the P-value. Count, the number of proteins. Size, the total number of genes in the pathway. KEGG, Kyoto Encyclopedia of Genes and Genomes.

framework for the design of specific drugs by identifying potential therapeutic targets $(13,23)$. The purpose of this study was to analyze the contribution of these proteins to the pathogenesis of PCa and discover other key proteins cooperating with them by topological analyses. To minimize the heterogeneity of the research subjects to the maximum extent, we selected proteomics studies in the literature focusing on human prostate tissue instead of biological fluids. On this basis, to further ensure the reliability of the DEPs in proteomics studies, we screened 41 PCa-associated DEPs that were reported more than 2 times from different researchers or further experimentally confirmed as seed proteins. The constructed network consisted of a giant network and 3 small separate components (Fig. 1). Only 3 seed proteins (AZGP1, CAPG and POSTN) were separate from the giant network, suggesting that the PPI between these proteins orchestrates the genesis of $\mathrm{PCa}$. It is possible that some proteins were missed in the literature search and new reliable proteins remain to be discovered for $\mathrm{PCa}$, in addition to false nodes resulting from false interactions in the network. However, biological networks are tolerant to the deletion of nodes, and new nodes prefer to link to nodes with a large degree. In other words, biological networks are robust to the random alteration of nodes, but are sensitive to hub removal $(10,22)$.

Ideally, a topological network analysis identifies proteins susceptible to be biomarkers or therapeutic targets (24). Because cancer-related proteins have a higher ratio of promiscuous 
structural domains, they are more prone to interact with other proteins. In fact, they have a large number of interacting proteins and occupy a central position in the networks (25). Proteins interacting with cancer-related proteins have a higher probability of being related to the cancer process than noninteracting proteins. Hence, the study of these proteins may be an efficient way to discover novel cancer genes and cancer biomarkers (26-28). In the giant network, TUBB2C is a hub protein with the largest degree and second highest $\mathrm{BC}$ value. Through topological analysis of the subnetwork, we found that TUBB2C also had the largest degree and the highest $B C$ value. TUBB2C is a member of the tubulin family. Tubulin, the major protein in microtubules, consists of a heterodimer of subunits designated as $\alpha$ and $\beta$. Both $\alpha$ - and $\beta$-tubulins are encoded by 6 to 7 genes each and exist as multiple isotypes in cells (29). TUBB2C is one of the $\beta$-tubulin isotypes; it is also known as $\beta 2$, TUBB 2 and TUBB4B. Currently, there are very few detailed studies on the association between TUBB4B and PCa. Only Ranganathan et al have proposed that the expression of the $\beta 2$ tubulin isotype in PCa and BPH tissues differs (30). However, the specific mechanisms of its involvement in $\mathrm{PCa}$ and subsequent research all lack specific elaboration. It has been found that TUBB2C is involved in the genesis and development of many types of tumors. The increased expression of TUBB2C is associated with the early lymph node micrometastasis of colorectal cancer (31); high levels of expression of TUBB2C in neuroepithelial tumors may reflect architectural changes in the developing brain (29). The downregulation of TUBB2C is also important in the development of nasopharyngeal carcinoma (32). ALB is a bottleneck protein with the highest BC value and second largest degree. Research has confirmed that a low preoperative serum albumin level may indicate extensive disease of clinically localized $\mathrm{PCa}$ and may ultimately be correlated with biochemical recurrence (33). In this study, TUBB2C and ALB were located in the top two of the $\mathrm{BC}$ and degree values in the giant network, suggesting that TUBB2C and ALB may be involved in the development and progression of PCa. However, further studies are required to verify our hypothesis.

In the backbone network, SLC2A4 has the largest degree and the highest $\mathrm{BC}$ value. In the giant network, backbone network and subnetwork, SLC2A4 was also located in the center with the highest $\mathrm{CC}$ value, suggesting that SLC2A4 may play an important role in the genesis of PCa. SLC2A4, also known as GLUT4, is a member of the glucose transporter (GLUT) family (34). It has been found that SLC2A4 can be used as a potential biomarker for many types of malignant tumors, including lung carcinoma, endometrial carcinoma, gastric cancer and breast cancer (35-38). Cancer cells need a steady source of metabolic energy to achieve uncontrolled growth and proliferation. Accelerated glycolysis is one of the biochemical characteristics of tumor cells, and the glycolytic breakdown of glucose is initiated by the transport of glucose, a rate-limiting process mediated by GLUT. Increased GLUT expression in malignant cells has been associated with the deregulated expression of GLUT proteins (39). GonzalezMenendez et al demonstrated that SLC2A4 is present in PCa cells and participates actively in glucose uptake. SLC2A4 is more important in glucose uptake in androgen-insensitive than in androgen-sensitive PCa cells (40). However, studies on SLC2A4 participating in PCa are relatively rare. This study found that SLC2A4 has an important node in the proteomics PPI network, and may act as a candidate for molecular markers and drug targeting associated with PCa.

To further confirm the role of TUBB2C and SLC2A4 in the giant network and backbone network, we constructed a subnetwork consisting of all of the shortest paths between the seed proteins (Fig. 4). The results revealed that TUBB2C had the highest BC value and largest degree. SLC2A4 was also located at the center of the subnetwork. Moreover, in this subnetwork, out of the 63 nodes with a high $\mathrm{BC}$ value in the giant network, only 3 seed proteins were excluded, indicating that the nodes with large BC values can efficiently connect and integrate these seed proteins. We also determined that the top $63 \mathrm{BC}$ nodes in this subnetwork coincided well with the 63 nodes with large $\mathrm{BC}$ value in the giant network.

Through module analysis, we found a densely connected region which contained the seed proteins, KRT8, KRT18 and HSPA5. KRT8 and $18(\mathrm{~K} 8 / 18)$ are simple epithelial cell-specific intermediate filament proteins. The loss of K8/18 expression during epithelial-mesenchymal transition (EMT) is associated with metastasis and chemoresistance (41). Fortier et al noted that K8/18 knockdown increases epithelial cancer cell motility and invasion without modulating EMT markers, and improves PI3K/Akt activation in epithelial cancer cells (41). HSPA5 plays a critical role in tumorigenesis, progression and resistance to chemotherapeutic agents. Approximately $70 \%$ of human PCa tumors express high levels of HSPA5, which is associated with recurrence, development of castration resistance and poor survival (42). Following Gene Ontology (GO) and KEGG pathway analysis, we found that the Ras protein signal transduction biological process and the MAPK signaling pathway were overrepresented. It has been suggested that Ras may be involved in the genesis and development of PCa. It has been shown that enhanced Ras signaling can reduce dependency for androgens in the LNCaP PCa cell line (43), whereas the inhibition of Ras can restore hormone dependency in $\mathrm{C} 42$ cells, a line that is otherwise hormone-independent $(43,44)$. Furthermore, as a downstream target of Ras signaling, MAPK levels are augmented in patients who have failed hormone ablation therapy (45). Finally, Ras activation in the DU145 human PCa cell line has been shown to promote metastasis to the brain and bones (46). Recently, Mulholland et al suggested that RAS/ MAPK pathway activation may serve as a potentiating second hit to PTEN/PI3K/AKT pathway alterations to androgendependent PCa and castrate-resistant PCa (47). These aforementioned data support the hypothesis that Ras protein signal transduction and the MAPK signaling pathway may act as molecular target candidates associated with PCa.

In conclusion, in the present study, we conducted meticulous collation and mining of proteomics studies in the literature on PCa and identified 41 DEPs between cancer and normal or benign tissues. We then adopted a systems biology method in order to construct an extended PPI network related to PCa. Through topological analysis of the giant network, backbone network and subnetwork, we identified SLC2A4 and TUBB2C as network biomarkers; however, further research is required to determine their function and mechanisms of action in PCa. In addition, by module analysis, we determined that Ras protein signal transduction and the MAPK signaling pathway may play an important role in the genesis and development of PCa. This 
study conducted a comprehensive analysis of the protein interaction network for proteomics DEPs in the overall perspective. Further investigations of these network biomarkers, biological process and pathways are warranted and this may reveal the specific pathogenesis of PCa and develop new targets for clinical treatments.

\section{Acknowledgements}

This study was supported by the National Natural Science Foundation of China (grant no. 81100518), the Science and Technology Foundation of Hebei Provincial Higher Education for Youth (grant no. QN2014013) and the Social Science Fund Project of Hebei Province (grant no. HB12SH030).

\section{References}

1. Siegel R, Ma J, Zou Z and Jemal A: Cancer statistics, 2014. CA Cancer J Clin 64: 9-29, 2014.

2. Smith RA, Manassaram-Baptiste D, Brooks D, Doroshenk M, Fedewa S, Saslow D, Brawley OW and Wender R: Cancer screening in the United States, 2015: A review of current American cancer society guidelines and current issues in cancer screening. CA Cancer J Clin 65: 30-54, 2015.

3. Davalieva K, Kiprijanovska S, Komina S, Petrusevska G, Zografska NC and Polenakovic M: Proteomics analysis of urine reveals acute phase response proteins as candidate diagnostic biomarkers for prostate cancer. Proteome Sci 13: 2, 2015.

4. Dihazi GH and Dihazi H: Protein-protein interaction networks improve the proteomics data interpretation in induced apoptosis Expert Rev Proteomics 7: 177-180, 2010.

5. von Mering C, Krause R, Snel B, Cornell M, Oliver SG, Fields S and Bork P: Comparative assessment of large-scale data sets of protein-protein interactions. Nature 417: 399-403, 2002.

6. Xin-Yu Y and Zheng-Ping X: An introduction to protein-protein interaction database and its application. Chin J Biochem Mol Biol 24: 189-196, 2008

7. Rakshit H, Rathi N and Roy D: Construction and analysis of the protein-protein interaction networks based on gene expression profiles of Parkinson's disease. PLoS One 9: e103047, 2014.

8. Sun J and Zhao Z: A comparative study of cancer proteins in the human protein-protein interaction network. BMC Genomics 11 (Suppl 3): S5, 2010.

9. Lee SA, Tsao TT, Yang KC, Lin H, Kuo YL, Hsu CH, Lee WK, Huang KC and Kao CY: Construction and analysis of the proteinprotein interaction networks for schizophrenia, bipolar disorder, and major depression. BMC Bioinformatics 12 (Suppl 13): S20, 2011.

10. Ran J, Li H, Fu J, Liu L, Xing Y, Li X, Shen H, Chen Y, Jiang X, $\mathrm{Li} \mathrm{Y}$, et al: Construction and analysis of the protein-protein interaction network related to essential hypertension. BMC Syst Biol 7: 32, 2013.

11. Davalieva K, Kostovska IM, Kiprijanovska S, Markoska K, Kubelka-Sabit K, Filipovski V, Stavridis S, Stankov O, Komina S, Petrusevska G and Polenakovic M: Proteomics analysis of malignant and benign prostate tissue by 2D DIGE/MS reveals new insights into proteins involved in prostate cancer. Prostate 75: $1586-1600,2015$

12. Wu CH, Yeh LS, Huang H, Arminski L, Castro-Alvear J, Chen Y, Hu Z, Kourtesis P, Ledley RS, Suzek BE, et al: The Protein Information Resource. Nucleic Acids Res 31: 345-347, 2003.

13. Sardiu ME and Washburn MP: Building protein-protein interaction networks with proteomics and informatics tools. J Biol Chem 286: 23645-23651, 2011.

14. Lee S-A, Chan C-H, Chen T-C, Yang CY, Huang KC, Tsai CH, Lai JM, Wang FS, Kao CY and Huang CY: POINeT: Protein interactome with sub-network analysis and hub prioritization. BMC Bioinformatics 10: 114, 2009.

15. Saito R, Smoot ME, Ono K, Ruscheinski J, Wang PL, Lotia S, Pico AR, Bader GD and Ideker T: A travel guide to Cytoscape plugins. Nat Methods 9: 1069-1076, 2012.

16. Jeanquartier F, Jean-Quartier C and Holzinger A: Integrated web visualizations for protein-protein interaction databases. BMC Bioinformatics 16: 195, 2015.
17. Raman K: Construction and analysis of protein-protein interaction networks. Autom Exp 2: 2, 2010.

18. Xie W, Sun J and Wu J: Construction and analysis of a proteinprotein interaction network related to self-renewal of mouse spermatogonial stem cells. Mol Biosyst 11: 835-843, 2015.

19. Scardoni G, Petterlini M and Laudanna C: Analyzing biological network parameters with CentiScaPe. Bioinformatics 25: 2857-2859, 2009

20. Rhrissorrakrai K and Gunsalus KC: MINE: Module identification in networks. BMC Bioinformatics 12: 192, 2011.

21. Dennis G Jr, Sherman BT, Hosack DA, Yang J, Gao W, Lane HC, Lempicki RA: DAVID: Database for annotation, visualization, and integrated discovery. Genome Biol 4: P3, 2003.

22. Lima-Mendez G and van Helden J: The powerful law of the power law and other myths in network biology. Mol Biosyst 5: 1482-1493, 2009.

23. Xia J, Benner MJ and Hancock RE: NetworkAnalyst - integrative approaches for protein-protein interaction network analysis and visual exploration. Nucleic Acids Res 42: W167-W174, 2014.

24. Sanz-Pamplona R, Berenguer A, Sole X, Cordero D, Crous-Bou M, Serra-Musach J, Guinó E, Pujana MÁ and Moreno V: Tools for protein-protein interaction network analysis in cancer research. Clin Transl Oncol 14: 3-14, 2012.

25. Jonsson PF and Bates PA: Global topological features of cancer proteins in the human interactome. Bioinformatics 22: 2291-2297, 2006.

26. Xu J and Li Y: Discovering disease-genes by topological features in human protein-protein interaction network. Bioinformatics 22: 2800-2805, 2006.

27. Sanz-Pamplona R, Aragüés R, Driouch K, Martín B, Oliva B, Gil M, Boluda S, Fernández PL, Martínez A, Moreno V, et al: Expression of endoplasmic reticulum stress proteins is a candidate marker of brain metastasis in both ErbB-2+ and ErbB-2 primary breast tumors. Am J Pathol 179: 564-579, 2011.

28. Pujana MA, Han JD, Starita LM, Stevens KN, Tewari M, Ahn JS, Rennert G, Moreno V, Kirchhoff T, Gold B, et al: Network modeling links breast cancer susceptibility and centrosome dysfunction. Nat Genet 39: 1338-1349, 2007.

29. Sugita Y, Nakamura Y, Yamamoto M, Oda E, Tokunaga O and Shigemori M: Expression of tubulin beta II in neuroepithelial tumors: Reflection of architectural changes in the developing human brain. Acta Neuropathol 110: 127-134, 2005.

30. Ranganathan S, Salazar H, Benetatos CA and Hudes GR: Immunohistochemical analysis of beta-tubulin isotypes in human prostate carcinoma and benign prostatic hypertrophy. Prostate 30: 263-268, 1997.

31. He ZY, Wen H, Shi CB and Wang J: Up-regulation of hnRNP A1, Ezrin, tubulin $\beta-2 \mathrm{C}$ and Annexin A1 in sentinel lymph nodes of colorectal cancer. World J Gastroenterol 16: 4670-4676, 2010.

32. Chan CML, Wong SCC, Lam MYY, Hui EP, Chan JK, Lo ES, Cheuk W, Wong MC, Tsao SW and Chan AT: Proteomic comparison of nasopharyngeal cancer cell lines C666-1 and NP69 identifies down-regulation of annexin II and $\beta 2$-tubulin for nasopharyngeal carcinoma. Arch Pathol Lab Med 132: 675-683, 2008.

33. Sejima T, Iwamoto H, Masago T, Morizane S, Yao A, Isoyama T, Kadowaki $\mathrm{H}$ and Takenaka A: Low pre-operative levels of serum albumin predict lymph node metastases and ultimately correlate with a biochemical recurrence of prostate cancer in radical prostatectomy patients. Cent European J Urol 66: 126-132, 2013.

34. Aparicio LM, Villaamil VM, Calvo MB, Rubira LV, Rois JM, Valladares-Ayerbes M, Campelo RG, Bolós MV and Pulido EG: Glucose transporter expression and the potential role of fructose in renal cell carcinoma: A correlation with pathological parameters. Mol Med Rep 3: 575-580, 2010.

35. Ito T, Noguchi $Y$, Satoh $S$, Hayashi $H$, Inayama $Y$ and Kitamura $H$ : Expression of facilitative glucose transporter isoforms in lung carcinomas: Its relation to histologic type, differentiation grade, and tumor stage. Mod Pathol 11: 437-443, 1998.

36. Shibata K, Kajiyama H, Ino K, Nawa A, Nomura S, Mizutani S and Kikkawa F: P-LAP/IRAP-induced cell proliferation and glucose uptake in endometrial carcinoma cells via insulin receptor signaling. BMC Cancer 7: 15, 2007.

37. Liu J, Wen D, Fang X, Wang X, Liu T and Zhu J: p38MAPK signaling enhances glycolysis through the up-regulation of the glucose transporter GLUT-4 in gastric cancer cells. Cell Physiol Biochem 36: 155-165, 2015.

38. Garrido P, Osorio FG, Morán J, Cabello E, Alonso A, Freije JM and González C: Loss of GLUT4 induces metabolic reprogramming and impairs viability of breast cancer cells. J Cell Physiol 230: 191-198, 2015. 
39. Won HJ, Ha TK, Kwon SJ, Cho HY, Hur SJ, Baik HH, Suh SI, Ha E and Kim YH: Differential effects of 5-fluorouracil on glucose transport and expressions of glucose transporter proteins in gastric cancer cells. Anticancer Drugs 21: 270-276, 2010.

40. Gonzalez-Menendez P, Hevia D, Rodriguez-Garcia A, Mayo JC and Sainz RM: Regulation of GLUT transporters by flavonoids in androgen-sensitive and -insensitive prostate cancer cells. Endocrinology 155: 3238-3250, 2014.

41. Fortier AM, Asselin E and Cadrin M: Keratin 8 and 18 loss in epithelial cancer cells increases collective cell migration and cisplatin sensitivity through claudin1 up-regulation. J Biol Chem 288: 11555-11571, 2013.

42. Misra UK and Pizzo SV: Ligation of cell surface GRP78 with antibody directed against the $\mathrm{COOH}$-terminal domain of GRP78 suppresses Ras/MAPK and PI 3-kinase/AKT signaling while promoting caspase activation in human prostate cancer cells. Cancer Biol Ther 9: 142-152, 2010.

43. Bakin RE, Gioeli D, Bissonette EA and Weber MJ: Attenuation of Ras signaling restores androgen sensitivity to hormone-refractory C4-2 prostate cancer cells. Cancer Res 63: 1975-1980, 2003.

44. Erlich S, Tal-Or P, Liebling R, Blum R, Karunagaran D, Kloog Y and Pinkas-Kramarski R: Ras inhibition results in growth arrest and death of androgen-dependent and androgen-independent prostate cancer cells. Biochem Pharmacol 72: 427-436, 2006

45. Jia S, Gao X, Lee SH, Maira SM, Wu X, Stack EC, Signoretti S, Loda M, Zhao JJ and Roberts TM: Opposing effects of androgen deprivation and targeted therapy on prostate cancer prevention. Cancer Discov 3: 44-51, 2013

46. Yin J, Pollock C, Tracy K, Chock M, Martin P, Oberst M and Kelly K: Activation of the RalGEF/Ral pathway promotes prostate cancer metastasis to bone. Mol Cell Biol 27: 7538-7550, 2007.

47. Mulholland DJ, Kobayashi N, Ruscetti M, Zhi A, Tran LM, Huang J, Gleave M and Wu H: Pten loss and RAS/MAPK activation cooperate to promote EMT and metastasis initiated from prostate cancer stem/progenitor cells. Cancer Res 72: 1878-1889, 2012.
48. Sun C, Song C, Ma Z, Xu K, Zhang Y, Jin H, Tong S, Ding W, Xia G and Ding Q: Periostin identified as a potential biomarker of prostate cancer by iTRAQ-proteomics analysis of prostate biopsy. Proteome Sci 9: 22, 2011

49. Pang J, Liu W-P, Liu X-P, Li LY, Fang YQ, Sun QP, Liu SJ, Li MT, Su ZL and Gao X: Profiling protein markers associated with lymph node metastasis in prostate cancer by DIGE-based proteomics analysis. J Proteome Res 9: 216-226, 2010.

50. Ummanni R, Junker H, Zimmermann U, Venz S, Teller S, Giebel J, Scharf C, Woenckhaus C, Dombrowski F and Walther R: Prohibitin identified by proteomic analysis of prostate biopsies distinguishes hyperplasia and cancer. Cancer Lett 266: 171-185, 2008.

51. Alaiya AA, Al-Mohanna M, Aslam M, Shinwari Z, Al-Mansouri L, Al-Rodayan M, Al-Eid M, Ahmad I, Hanash K, Tulbah A, et al: Proteomics-based signature for human benign prostate hyperplasia and prostate adenocarcinoma. Int $\mathbf{J}$ Oncol 38: 1047-1057, 2011.

52. Geisler C, Gaisa NT, Pfister D, Fuessel S, Kristiansen G, Braunschweig T, Gostek S, Beine B, Diehl HC, Jackson AM, et al: Identification and validation of potential new biomarkers for prostate cancer diagnosis and prognosis using 2D-DIGE and MS. BioMed Res Int 2015: 454256, 2015

53. Skvortsov S, Schäfer G, Stasyk T, Fuchsberger C, Bonn GK, Bartsch G, Klocker $\mathrm{H}$ and Huber LA: Proteomics profiling of microdissected low- and high-grade prostate tumors identifies Lamin A as a discriminatory biomarker. J Proteome Res 10: 259-268, 2011.

54. Han ZD, Zhang YQ, He HC, Dai QS, Qin GQ, Chen JH, Cai C, Fu X, Bi XC, Zhu JG, et al: Identification of novel serological tumor markers for human prostate cancer using integrative transcriptome and proteome analysis. Med Oncol 29: 2877-2888, 2012. 